DOI: 10.1002/adma.((please add manuscript number))

\title{
« Hyper-bright» Near-Infrared Emitting Fluorescent Organic Nanoparticles for Single Particle Tracking
}

Emilie Genin, Zhenghong Gao, Juan A. Varela, Jonathan Daniel, Talia Bsaibess, Isabelle Gosse, Laurent Groc, Laurent Cognet, * Mireille Blanchard-Desce*

Dr. Emilie Genin, Jonathan Daniel, Talia Bsaibess, Dr. Isabelle Gosse, Dr. Mireille

Blanchard-Desce

Univ. Bordeaux, Institut des Sciences Moléculaires (UMR 5255), 351 Cours de la Libération, 33405 Talence, France

E-mail: mireille.blanchard-desce@u-bordeaux1.fr

Dr. Juan A. Varela, Dr. Laurent Groc

Interdisciplinary Institute for Neuroscience (UMR 5297), Univ. Bordeaux, 33000 Bordeaux, France

Dr. Laurent Cognet, Dr. Zhenghong Gao

LP2N, Univ. Bordeaux, UMR 5298, 351 cours de la libération, F-33405 Talence, France; Institut d'Optique \& CNRS, UMR 5298, F-33405 Talence, France

E-mail: lcognet@u-bordeaux1.fr

Keywords: luminescence, biocompatibility, photostability, single NP, imaging in cellular environment

Single molecule imaging is powerful tool for revealing the dynamic behavior of molecules in complex environments. In general, imaging and tracking a biomolecule of interest requires labeling it with a marker giving optical contrast to be detected by an optical microscope. Common markers can be luminescent ${ }^{[1]}$ or non luminescent ${ }^{[2]}$ nano-objects and can allow single particle tracking (SPT) the motion of biomolecules both for in vivo and in vitro applications. Ideally, optical probes should be bright, stable in physiological and cellular environments, have large photostability with low toxicity and good biocompatibility (i.e. do not disturb or destroy healthy cells). Small aromatic organic fluorophores ${ }^{[3]}$ and autofluorescent proteins have been the earliest single molecule fluorescent probes used in living cells. ${ }^{[4]}$ They can generate bright fluorescence, but most of the time they experience fast photobleaching and photo-blinking and are thereby limited to short time imaging. Inorganic 
are popular probes due to their high luminescent nanoparticles, such as quantum dots, are popular probes due to their high brightness and photostability, ${ }^{[1]}$ but raise biodegradability issues. In addition, all these probes commonly have emission in the visible range, where the luminescence signal can be readily obscured by the background fluorescence from organisms (i.e. originating from endogenous chromophores). Actually, the optimal imaging spectral window (both for excitation and emission) lies in the near Infra-red (NIR) region, where biological samples are the most transparent. In that context, the search and synthesis of bright, biocompatible nanoparticles with NIR emission appears of major importance for the development of high-performance sensors. NIR-emitting inorganic quantum dots are one option; however, they commonly contain heavy metals and thereby show toxicity, resulting in cells death. ${ }^{[5]}$ Single wall carbon nanotubes are another promising option but still require extensive synthesis and preparation steps to assess biocompatibility. ${ }^{[6]}$ Fluorescent organic nanoparticles (FONs) have advantages such as their ease of preparation, reduced cost, as well as their high versatility, and possible degradability and possible reduced toxicity (depending on their organic components). ${ }^{[7]}$ They have motivated numerous studies for lighting and imaging purposes. ${ }^{[8]}$ However, the synthesis of bright and biocompatible FONs with stable NIR emission over time and compatible with physiological conditions is still in its infancy. This is due to the relative scarcity of NIRemitting dyes suitable for the elaboration of FONs and retaining NIR luminescence in water. Most molecular NIR emitters such as cyanine dyes include an extended planar delocalized $\pi$ system, favorable to NIR luminescence of isolated molecules, but which favors $\pi-\pi$ stacking in aggregates or crystals. Such intermolecular interactions may result in fluorescence quenching, calling for subtle molecular engineering strategies to be implemented to prevent molecular packing deleterious to fluorescence. Moreover intermolecular excited-state processes such as energy and electron transfer enabled in close-packed assemblies, can lead to fluorescence quenching. Hence, the design of high-performance NIR emitting dyes dedicated 


\section{Submitted to}

to the elaboration of FONs retaining NIR emission in water and having robust structures and stable physicochemical properties (including in biological environments) remains challenging. In this communication, we present our approach to specially-engineered synthetic NIR emitting dye allowing the fast preparation of hyperbright, NIR-emitting FONs, which we named HIFONs. These innovative fully-organic nanoparticules are shown to exhibit excellent chemical and colloidal stability as well as robust luminescence (also in cell environments) and good photostability and biocompatibility. Thanks to the exceptional combination of these specific features, the HIFONs could be used for single NP imaging in bioenvironments as well as for SPT in water.

Our strategy was based on tailored "push-pull" chromophores built from an electrondonating group (D) connected via a $\pi$-conjugated system to an electron-withdrawing group (A). ${ }^{[7]}$ Such chromophores display intense, low-energy intramolecular charge transfer (ICT) transition in the near UV-visible range depending on the nature of the D/A pair and conjugated system. In particular suitable combination allows to tune the ground-state structure and polarization close to the "cyanine-limit" where the electron are delocalized along the conjugated system and the energy gap is minimum while the transition dipole is maximum. ${ }^{[9]}$ These characteristics result in both intense and red-shifted excitation bands. In addition, the electronic delocalization results in lower conformational flexibility of the conjugated $\pi$ system which is favorable to luminescence as some of the vibrational deactivation processes are repressed. In order to shift the excitation (and emission) to red and NIR region, a powerful strategy is to increase the $\pi$-conjugated system. ${ }^{[10]}$ Our choice was to focus on push-pull chromophores built from an oligothiophene $\pi$-conjugated system (Figure 1) since this connector allows efficient electronic communication, which leads to large radiative decay rates, while limiting non-radiative decay compared to polyenes (due to lower degrees of conformational flexibility) thus leading to luminescent compounds. ${ }^{[11]}$ We selected a pair of 

strong donor $(\mathrm{D}=$ aminophenyl) and acceptor $(\mathrm{A}=$ diethylthiobarbituric) groups so as to favor strong ICT and electron delocalization. ${ }^{[10]}$ As large ground-state polarization is expected from design, resulting in large electrostatic (dipole-dipole) interactions in condensed phases, additional molecular engineering was required to prevent antiparallel association (which is favored in the case of rod-like chromophores) and subsequent fluorescence quenching. ${ }^{[12]}$ In order to hinder the formation of tight antiparallel dimers, two tbutyl-biphenyl-substituents were grafted on the donating nitrogen atom (Scheme 1) which act as fins, shifting the overall shape of the push-pull structure from a rod-like to a three-dimensional one (Figure 1). These substituents are also conveying increased solubility of chromophore $\mathbf{3}$ in organic solvents, while decreasing its solubility in pure water. This difference in solubility is critical for the easy and rapid production of organic nanoparticles using the simple reprecipitation process. ${ }^{[13]}$

The synthesis of dye $\mathbf{3}$ was easily achieved starting from aldehyde $\mathbf{1}$ by means of a three-step sequence involving a bromination reaction followed by a Suzuki-Miyaura crosscoupling and a Knoevenagel condensation (Scheme 1). Dye 3 was obtained with $52 \%$ overall yield as a pure dark solid which was fully characterized by ${ }^{1} \mathrm{H}$ and ${ }^{13} \mathrm{C}$ NMR spectroscopies, elemental analysis and mass spectrometric techniques. The experimental procedures details and related compounds characterizations are reported in the Experimental Section. Dye 3 shows intense absorption bands located in the UV and visible region when dissolved in organic solvents (see Figure S1) and a definite fluorescence emission varying from orange to red or NIR depending on the polarity of its environment (see Figure S2). FONs made from dye 3 were easily obtained by fast addition of a mM solution of $\mathbf{3}$ in THF into pure water at room temperature, leading almost instantaneously to limpid and bright NIR-emitting $\left(\lambda_{\mathrm{em}}{ }^{\max }=800 \mathrm{~nm}\right)$ fluorescent organic nanoparticles suspensions (HIFONs) in water. The formation of organic nanoparticles was further attested by Transmission Electron Microscopy (TEM) which was used to evaluate the morphology and size distribution of the FONs. TEM images showed that FONs were mainly spherical (Figure 1). The size distribution is close to a 

unimodal distribution centered on $40 \mathrm{~nm}$ with a FWHM of $22 \mathrm{~nm}$, indicating a narrow and homogeneous distribution of the diameters (See Figure S3). The sizes obtained from TEM observations were in a very good agreement with diameters obtained from Dynamic Light Scattering (DLS) which yields an average hydrated diameter of $53 \mathrm{~nm}(\mathrm{PdI}=0.19)$. All these observations suggested that FONs had uniform nearly spherical and homogeneous dimensions. Interestingly, both the chemical and colloidal stability of HIFONs were found to be remarkable. Whereas dye 3, which is obtained from a Knoevenagel condensation, is observed to undergo retro-Knoevenagel reaction when dissolved in polar organic solvents containing water traces (such as acetone or DMSO, Figure S5), such a reaction is suppressed in HIFONs in water (see Figure S4). This demonstrates the absence of reactivity of surface dyes in HIFONs with surrounding water (or exchange with bulk), emphasizing that the chemical integrity of the HIFONs is preserved over time. Furthermore, the colloidal stability is found to be remarkable with morphology and size remaining stable after several days. Importantly the absorption or photoluminescence characteristics of FONs suspension in water were found to be stable over long period of times (see Figures S4), indicating that HIFONs made from dye 3 retain both their chemical structure and morphological features but also their giant brightness (with an estimated value of $2.10^{7} \mathrm{M}^{-1} \mathrm{~cm}^{-1}$ in deionized water) over time. Indeed this brightness is about 3 to 4 orders of magnitude larger than those of prototypical water-soluble NIR emitting organic dyes (such as cyanine or ATTO dyes, see Table S2), validating the appellation HIFONs. Interestingly the HIFONs are found to be stable in different $\mathrm{pH}$ conditions (Figures S6) and their luminescence is found to be enhanced in the presence of PBS buffer (see Figure S7).

Taking advantage of the robustness of the newly obtained HIFONS, we next studied their photoluminescence (PL) properties. We employed a home-made wide-field fluorescence microscope to image the FONs in different aqueous suspensions. HIFONs were diluted either in water or PBS $1 \mathrm{X}$ buffer to prepare an aqueous HIFONs suspension $(\sim 50 \mathrm{pM})$ for single 


\section{Submitted to

particle observations. A drop $(\sim 20 \mu \mathrm{L})$ of solutions was sandwiched between two glass slides and mounted on the optical microscope. PL images showed that individual FONs could be detected in both media when excited at $568 \mathrm{~nm}$ with a laser. This establishes that HIFONs have stable structures and PL properties in water as well as in highly salty environments. In both cases, HIFONs displayed bright signals and no evidence of aggregation occurring during imaging. Figure 2 presents the PL intensity distribution measured on 521 individual FONs. Consistently with TEM analysis, the PL intensity distribution is close to a unimodal distribution centered on 1100 counts with a FWHM of 870 counts, indicating again a narrow and homogeneous distribution of the FON diameters with uniform PL properties in aqueous phases. Under these excitation conditions, individual HIFONs displayed a bi-exponential photobleaching decay behavior, with typical 1/e times of $\sim 6 \mathrm{~s}(50 \%)$ and $\sim 40 \mathrm{~s}(50 \%)$. This behavior likely originates from the combined effect of individual dye photophysics and multidye interactions within HIFONs. Owing to their bright emission, individual HIFONs can be easily detected for more than $25 s$ (Figure 2) while imaging time of a single NIR emitting organic dye (such as cyanine dyes) is limited to typically a few seconds. ${ }^{[1 \mathrm{a}]}$ This good signal stability allowed us to track the movement of individual HIFONs in water, which after an automated trajectory reconstruction process indicated again a monodisperse size distribution, as revealed by the diffusion coefficients histogram corresponding to the analyzed Brownian movements (Figure 2). The possibility of detecting individual HIFONs in a fluorescence microscope makes them real candidates to use as labels for single molecule studies in biological systems. The stability and toxicity of the HIFONs in biological environment was hence tested by incubating fibroblasts with HIFONs dispersed in cellular growth medium. As illustrated in Figure 2, HIFONs are internalized and retain their remarkable stability and PL luminescence in cell environment (even after hours). To further attest their bicompatibility, we performed two viability assays evaluating viable mitochondria and plasma membrane 
permeability, yielding no toxic effects of HIFONs for the tested concentration and incubation times (see Figure S8).

In conclusion, we have presented the design and chemical synthesis of a new type of chromophore $\mathbf{3}$ to achieve NIR emission as nano-aggregates. The PL of $\mathbf{3}$ interestingly changed from 600 to $900 \mathrm{~nm}$ due to the bipolar interactions with different surrounding solvents. Surprisingly, hyper-bright near IR-emitting fluorescent organic nanoparticles (which we thus called HIFONS) were easily obtained via a very simple one-pot strategy. The strategy was carried out and finished in a short time at room temperature. The produced HIFONs had stable structure and PL properties at various aqueous environments and show outstanding NIR brightness $\left(2.10^{7} \mathrm{M}^{-1} \mathrm{~cm}^{-1}\right.$ with $\left.\lambda_{\mathrm{em}}=800 \mathrm{~nm}\right)$, typically 3 orders of magnitude larger than those of prototypical water-soluble NIR emitting dyes . Moreover, FONs were successfully imaged at single particles level after 24 hour integrating into cellular environments. In perspective, upon imparting recognition features to HIFONs through suitable chemical functionalization, this novel nanoparticules are promising to be targeted to various receptors and proteins, as well as to DNA, and RNA, both in vivo and in vitro to reveal the dynamic secrets of biomolecules in NIR range by single particles imaging and tracking in the future. This opens a challenging route towards selective and effective functionalization of fully molecular-based HIFONs.

\section{Supporting Information}

Supporting Information is available online from the Wiley Online Library.

\section{Acknowledgements}

MBD gratefully acknowledges Conseil Régional d'Aquitaire for financial support (Chaire d'Accueil grant) and Univ. Bordeaux 1 for a fellowship to TB. LG, JV, LC, ZG are supported by CNRS, Conseil Régional d'Aquitaine and Agence Nationale de la Recherche. JV is supported by Marie Curie Training Program. We thank the Bordeaux Imaging CenterImagerie Electronique where TEM analysis was performed. 
Submitted to

\section{ADAANCED}

Note: Zhenghong Gao, Juan A. Varela and Jonathan Daniel contributed equally to the work.

[1] a) L. Groc, M. Lafourcade, M. Heine, M. Renner, V. Racine, J. B. Sibarita, B. Lounis, D. Choquet, L. Cognet, J. Neurosci. 2007, 27, 12433; b) F. Pinaud, S. Clarke, A. Sittner, M. Dahan, Nature methods 2010, 7, 275.

[2] L. Cognet, S. Berciaud, D. Lasne, B. Lounis, Anal. Chem. 2008, 80, 2288.

[3] a) Y. Sako, S. Minoghchi, T. Yanagida, Nat. Cell Biol. 2000, 2, 168; b) C. Tardin, L. Cognet, C. Bats, B. Lounis, D. Choquet, EMBO J. 2003, 22, 4656.

[4] G. S. Harms, L. Cognet, P. H. Lommerse, G. A. Blab, T. Schmidt, Biophys J 2001, 80, 2396.

[5] R. Hardman, Environ Health Perspect 2006, 114, 165.

[6] a) P. Cherukuri, S. M. Bachilo, S. H. Litovsky, R. B. Weisman, J Am Chem Soc 2004, 126, 15638; b) J. G. Duque, L. Cognet, A. N. G. Parra-Vasquez, N. Nicholas, H. K. Schmidt, M. Pasquali, J Am Chem Soc 2008, 130, 2626; c) K. Welsher, Z. Liu, S. P. Sherlock, J. T. Robinson, Z. Chen, D. Daranciang, H. Dai, Nat Nano 2009, 4, 773.

[7] V. Parthasarathy, S. Fery-Forgues, E. Campioli, G. Recher, F. Terenziani, M. Blanchard-Desce, Small 2011, 7, 3219.

[8] A. Patra, C. G. Chandaluri, T. P. Radhakrishnan, Nanoscale 2012, 4, 343 and references cited therein.

[9] a) M. Barzoukas, C. Runser, A. Fort, M. Blanchard-Desce, Chem. Phys. Lett. 1996, 257, 531; b) A. Painelli, F. Terenziani, J. Phys. Chem. A 2000, 104, 11041.

[10] M. Blanchard-Desce, V. Alain, P. V. Bedworth, S. R. Marder, A. Fort, C. Runser, M. Barzoukas, S. Lebus, R. Wortmann, Chem. Eur. J. 1997, 3, 1091.

[11] a) G. Barbarella, M. Zambianchi, O. Pudova, V. Paladini, A. Ventola, F. Cipriani, G. Gigli, R. Cingolani, G. Citro, J. Am. Chem. Soc. 2001, 123, 11600; b) G. Barbarella, M. Melucci, G. Sotgiu, Adv. Mater. 2005, 17, 1581; c) G. Ridolfi, N. Camaioni, P. Samori, M. Gazzano, G. Accorsi, N. Armaroli, L. Favaretto, G. Barbarella, J. Mater. Chem. 2005, 15, 895; d) M. Duca, B. Dozza, E. Lucarelli, S. Santi, A. Di Giorgio, G. Barbarella, Chem. Comm. 2010, 46, 7948; e) I. Palama, F. Di Maria, I. Viola, E. Fabiano, G. Gigli, C. Bettini, G. Barbarella, J. Am. Chem. Soc. 2011, 133, 17777; f) M. L. Capobianco, G. Barbarella, A. Manetto, Molecules 2012, 17, 910.

[12] F. Terenziani, M. Morone, S. Gmouh, M. Blanchard-Desce, ChemPhysChem 2006, 7, 685.

[13] H. Kasai, H. S. Nalwa, H. Oikawa, S. Okada, H. Matsuda, N. Minami, A. Kakuta, K. Ono, A. Mukoh, H. Nakanishi, Jpn. J. Appl. Phys. 1992, 31, L1132. 


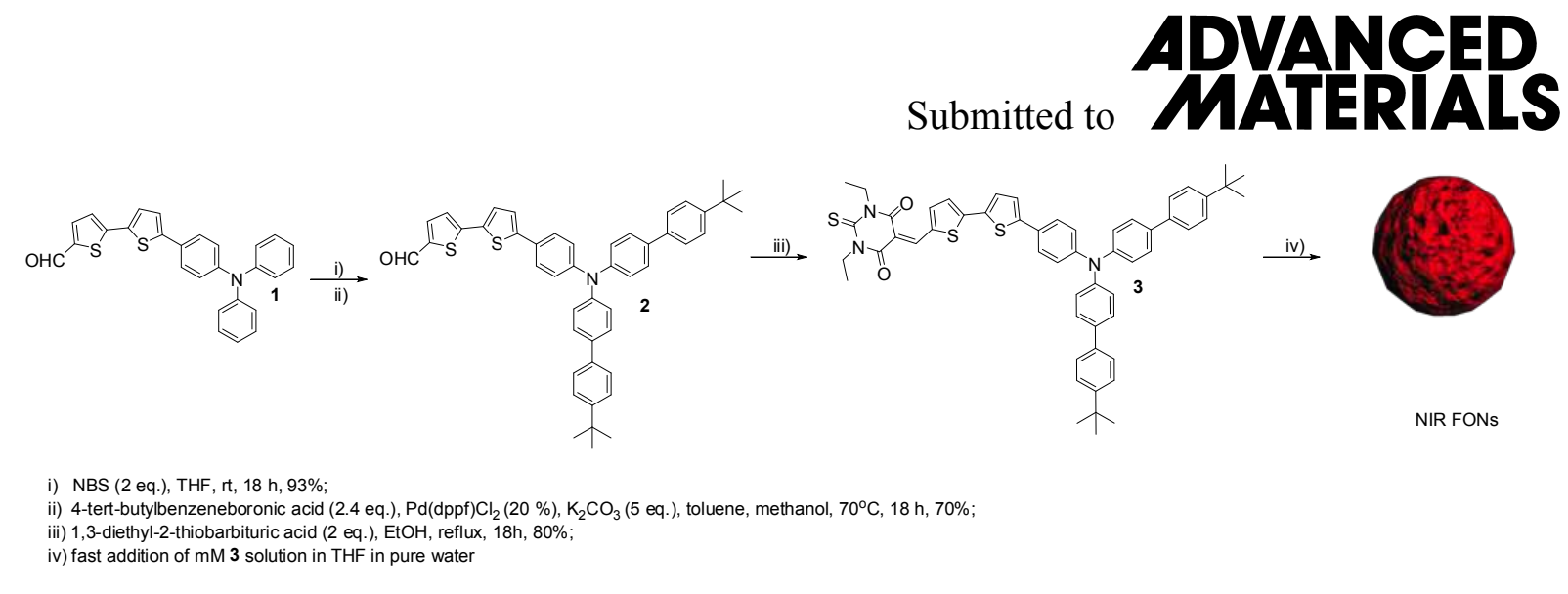

Scheme 1. Multi-step synthesis of chromophore 3 and subsequent one-pot synthesis of HIFONs by reprecipitation process.

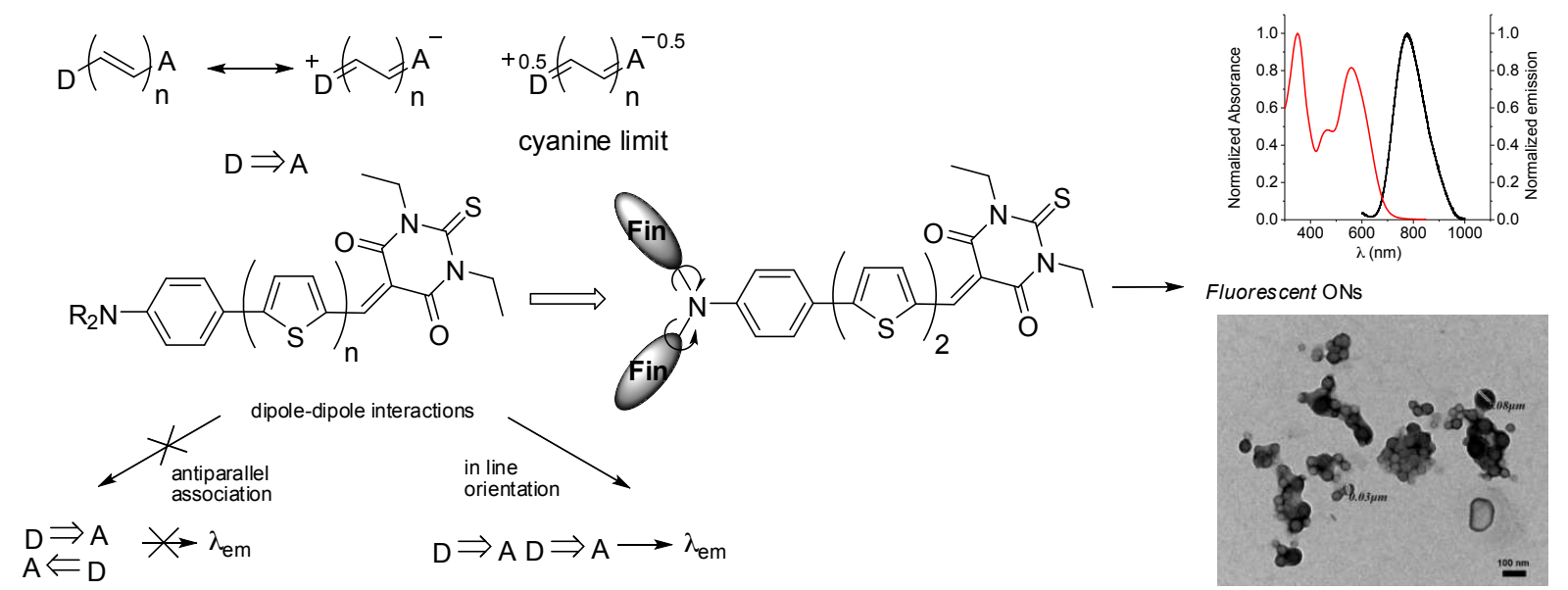

Figure 1. left: Molecular engineering of NIR chromophore 3 for the elaboration of hyperbright near IR emitting FONs (HIFONS), right: absorption and emission spectra of HIFONs made from 3 in water suspension and typical TEM image. 


\section{Submitted to
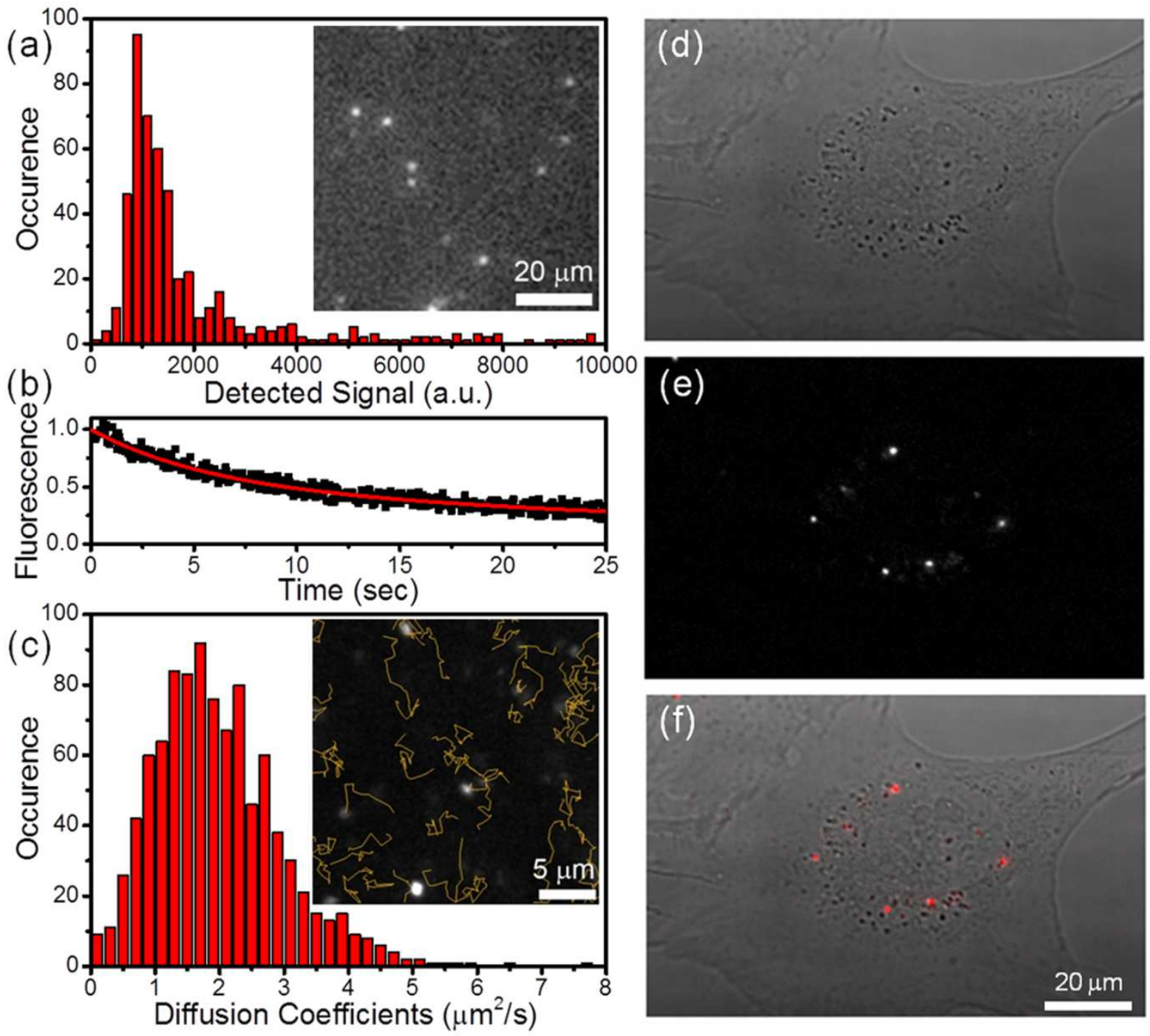

Figure 2. Left column: fluorescence properties and tracking of single HIFONs. (a) the distribution of fluorescence signals of HIFONs, inset shows a typical wide-field fluorescence image; (b) the photobleaching behavior of a single HIFON, black squares present normalized fluorescence, red line presents fitted fluorescence by a bi-exponential function; (c) the distribution of diffusion coefficients of HIFONs, inset shows the trajectories of HIFONs in water. Right column: fluorescence imaging of single HIFONs cultured with living mouse embryonic fibroblasts for $24 \mathrm{~h}$. (d) Bright field image, (e) wide-field fluorescence, and (f) the merged image. 